Proceedings of the 2011 Winter Simulation Conference

S. Jain, R.R. Creasey, J. Himmelspach, K.P. White, and M. Fu, eds.

\title{
AN APPLICATION OF FACTORIAL DESIGN TO COMPARE THE RELATIVE EFFECTIVENESS OF HOSPITAL INFECTION CONTROL MEASURES
}

\author{
Sean Barnes \\ Department of Mathematics \\ University of Maryland \\ College Park, MD, USA \\ Edward Wasil \\ Kogod School of Business \\ American University \\ Washington, DC, USA
}

\author{
Bruce Golden \\ Robert H. Smith School of Business \\ University of Maryland \\ College Park, MD, USA \\ Jon Furuno \\ Anthony Harris \\ Department of Epidemiology and Public Health \\ University of Maryland School of Medicine \\ Baltimore, MD, USA
}

\begin{abstract}
Optimal methods to control patient-to-patient transmission of methicillin-resistant Staphylococcus aureus (MRSA) in an intensive care unit (ICU) setting are still unknown. We iteratively applied a full $2^{k}$ factorial design on the output of a stochastic, agent-based simulation to compare the effects of the hand hygiene compliance of healthcare workers and the nurse-to-patient ratio on the transmission of MRSA in a 20-bed ICU. The results suggest that increasing the nurse-to-patient ratio is more effective at levels below approximately $60 \%$ compliance of nurses. However, improving the hand washing compliance of nurses becomes the better strategy at higher baseline compliance levels. In addition, interaction effects between the two infection control measures limit the marginal benefit of improving both factors to high levels.
\end{abstract}

\section{INTRODUCTION}

Infections with MRSA are a major problem among hospitalized patients (Cosgrove et al. 2005; Moland et al. 2006). Interventions have been proposed to decrease the transmission of MRSA in high-risk healthcare settings such as the intensive care unit (Weber et al. 2007; Muto et al. 2003). Two key factors that have been identified as effective infection control measures are improving the hand hygiene compliance of healthcare workers and increasing the ratio of nurses to patients in the hospital.

Hand hygiene compliance is a cornerstone of infection control. Many studies have attempted to demonstrate that improving hand hygiene compliance leads to fewer hospital-acquired infections. Pittet et al. (2000) demonstrated that the introduction of alcohol-based hand disinfectant, which likely caused an increase in hand hygiene compliance, reduced the incidence of MRSA infections. However, there are no experimental studies that have assessed the effect of improving hand hygiene compliance on MRSA colonization acquisition rates.

Improving nurse-to-patient ratios has also been advocated as a potentially effective intervention at curbing the transmission of antibiotic-resistant bacteria. This intervention should lead to fewer MRSA acquisitions by limiting the number of contacts between nurses and patients, thereby decreasing the opportunity to spread MRSA to multiple patients. Fridkin et al. (1996) reported that the reduction of the nursing staff below a critical level may contribute to an increase in catheter-associated bloodstream infec- 
tions. However, there are no experimental studies that have assessed the effect of changing the nurse-topatient ratio on MRSA acquisition rates.

Hospitals and resident epidemiologists often have limited resources and must choose between several potentially viable interventions aimed at decreasing patient-to-patient transmission of antibiotic-resistant bacteria such as MRSA. Mathematical modeling and simulation can be used to assess the potential benefits of different interventions when experimental trials have not been performed or cannot be performed due to ethical considerations (Fridkin et al. 1996; McKenzie and Samba 2004; Antia and Lipsitch 1997). There are several studies that have used mathematical modeling and computer simulation to evaluate the effectiveness of one or both of these factors in reducing the incidence of hospital-acquired infections (Austin et al. 1999; Beggs et al. 2006; Beggs, Shepherd, and Kerr 2008; Grundmann et al. 2002; Raboud et al. 2005; Sebille, Chevret, and Valleron 1997). These studies have compared the effectiveness of bundled interventions on MRSA acquisition for a particular hospital configuration, but have provided little or no consideration as to when, or in what cases, one intervention is better than another. In addition, these studies have not fully characterized how changing the level of one factor affects the effectiveness of the other factors. These interaction effects are a strong determinant of how much transmission can be reduced by improving a limited set of control measures. In this study, we compared the relative effectiveness of increasing the hand hygiene compliance of nurses and increasing the nurse-to-patient ratio to help inform hospitals and hospital epidemiologists considering these interventions.

\section{METHODOLOGY}

We use a stochastic, agent-based model developed by Barnes, Golden, and Wasil (2010) to simulate a 20bed ICU and the transmission of MRSA among patients through direct contact with healthcare workers. The agent-based formulation of the model facilitates the separation of behavior between patients, nurses, and physicians, so that their individual effects on the entire system can be evaluated. In addition, within each agent class, each individual can have unique characteristics and behavior, which provides more flexibility to analyze the system in detail.

\subsection{Model Assumptions and Parameter Estimates}

The model simulates patients entering the ICU, occupying a single room, and being discharged after their stays are completed. Each patient is visited a constant total number of times each day, but the relative proportion of visits by nurses and physicians can vary. During these visits, patients are susceptible to acquiring MRSA from transiently colonized healthcare workers. After each visit to a patient, a healthcare worker washes his or her hands with a given probability and a specified efficacy of removing MRSA. A healthcare worker removes any MRSA bacteria that may have been transiently acquired from a colonized patient if the hand washing event is successful, and he or she does not pose an immediate threat to transmitting MRSA to other patients. If hand washing is omitted or the event is unsuccessful, the healthcare worker can potentially transmit MRSA to other patients during subsequent visits.

In order to assess the relative effectiveness of improving hand hygiene compliance among nurses and increasing the nurse-to-patient ratio, we assumed that both the number of physicians and their hand hygiene behavior was constant (i.e., only parameters related to nurses were varied). Two physicians were responsible for 10 patients each in the ICU, and physician compliance was held constant at $65 \%$ throughout the experiments. The other key parameters for the model are summarized in Table 1. Given a set of input parameters, we simulate the interactions between patients and healthcare workers over a specified time period, and produce statistics on the number of transmissions to patient by nurses and physicians.

\subsection{Analytical Methods}

A full $2^{k}$ factorial design is applied to compare the effect of improving both the hand hygiene compliance of nurses and the nurse-to-patient ratio to determine which control measure has a larger impact on reducing MRSA transmission. A $2^{k}$ factorial design specifies two levels for each of the $k$ factors used in the 
Barnes, Golden, Wasil, Furuno, and Harris

Table 1: Model parameters and values

\begin{tabular}{|c|c|c|}
\hline Parameter & Value & Reference(s) \\
\hline Number of physicians & $2(1: 10$ ratio $)$ & \\
\hline Physician hand hygiene compliance & 0.65 & $\begin{array}{l}\text { Cromer et al. } 2008 \text {; } \\
\text { Duggan et al. } 2008\end{array}$ \\
\hline Hand hygiene efficacy & 0.95 & $\begin{array}{l}\text { Ojajarvi 1980; } \\
\text { Girou et al. } 1980\end{array}$ \\
\hline $\begin{array}{r}\text { Proportion of admitted patients } \\
\text { positive with MRSA }\end{array}$ & 0.10 & $\begin{array}{l}\text { Warren et al. 2006; } \\
\text { Ridenour et al. 2007; } \\
\text { Honda et al. } 2010\end{array}$ \\
\hline $\begin{array}{l}\text { Transmission probability from } \\
\text { patient to healthcare worker }\end{array}$ & 0.20 & \\
\hline $\begin{array}{l}\text { Transmission probability from } \\
\text { healthcare worker to patient }\end{array}$ & 0.05 & \\
\hline Patient length of stay & $\log \mathrm{N}(0.693,1.1646)^{*}$ & $\begin{array}{l}\text { Iwashyna, Kramer, } \\
\text { and Kahn 2009; Angus } \\
\text { et al. 1996. }\end{array}$ \\
\hline Visits per day per patient & 48 & $\begin{array}{l}\text { Kirkland and Wein- } \\
\text { stein } 1999\end{array}$ \\
\hline Proportion of patient visits by nurses & 0.90 & \\
\hline
\end{tabular}

$* \log \mathrm{N}(\alpha, \beta)$ represents the lognormal distribution with scale parameter $\alpha$ and shift parameter $\beta$. The parameters $\alpha$ and $\beta$ were determined to generate a distribution with a mean length of stay of 3.94 days and a median length of stay of 2 days.

experiment: a plus-level representing the factor value that has the better effect on the response and a minus-level that has a less desirable effect. Simulations are then conducted using the parameter values specified by each design point to generate a sample of the system response at that level. A full design simulates all factor-level combinations, whereas a partial design would use only a subset of the design points to evaluate the effects of the various factors. The two input factors for this study are the hand hygiene compliance of nurses and the nurse-to-patient ratio in the ICU. The system response is the number of MRSA acquisitions in the ICU over the simulation period. A sample factorial design is shown in Table 2.

Table 2: Sample factorial design

\begin{tabular}{cccc}
\hline $\begin{array}{c}\text { Design } \\
\text { Point }\end{array}$ & Factor A & Factor B & Response \\
\hline 1 & - & - & $R_{1}$ \\
2 & - & + & $R_{2}$ \\
3 & + & - & $R_{3}$ \\
4 & + & + & $R_{4}$ \\
\hline
\end{tabular}

There are two primary results derived from a factorial experiment. The first result is the set of main effects, each of which represents the average effect on the response by increasing one factor from its minus-level to its plus-level. Main effects $\left(e_{k}\right)$ can have positive or negative values, which respectively represent increasing or decreasing the response of the system as the factor level changes from minus to 
plus. Main effects that are close to zero suggest the factor has little to no effect on the system. The second result is the set of interaction effects between all unique subsets of two or more factors. Interaction effects $\left(e_{k_{1} k_{2}}\right)$ characterize how changing one factor affects the ability of the other factors in the chosen subset to influence the response. Small interaction effects imply that improving multiple factors simultaneously has nearly the same effect as adding together the benefits of improving each factor by itself, which is the maximum potential improvement. When interaction effects are large, other factors are less likely to have a significant effect on the response when one factor is at its plus-level. Interaction effects can be difficult to interpret, because they only provide a relative measure for how dependent one factor is on other factors. Equations to calculate the main effect of two factors and the interaction effect between them are shown in Equations (1), (2), and (3).

$$
\begin{aligned}
& e_{A}=\frac{-R_{1}-R_{2}+R_{3}+R_{4}}{2} \\
& e_{B}=\frac{-R_{1}+R_{2}-R_{3}+R_{4}}{2} \\
& e_{A B}=\frac{R_{1}-R_{2}-R_{3}+R_{4}}{2}
\end{aligned}
$$

We examine whether increasing the hand hygiene compliance of nurses from $0 \%$ to $100 \%$ in various increments had a better effect on MRSA acquisition rates than increasing the nurse-to-patient ratio from 1:4 to 1:1 in various increments. Rather than determine a single measure of effectiveness for each measure, we iteratively apply a $2 \times 2$ factorial design across the entire parameter space. Beginning at $0 \%$ compliance and a nurse-to-patient ratio of 1:4, we compute the mean main effects and mean interaction effect across 25 Monte Carlo replications over selected factor-level combinations, using the number of MRSA acquisitions as the response. We use increments of $5 \%, 10 \%, 15 \%, 20 \%$, and $25 \%$ between the plus- and minus-levels for the hand hygiene compliance of nurses and increases of one (e.g., 1:4 to 1:3) and two levels (e.g., 1:4 to 1:2) for the nurse-to-patient ratio. By computing the main and interaction effects across all selected factor-level combinations, we are able to gain a better understanding of where in the parameter space each factor has an advantage.

There is one issue that arises when comparing interaction effects across the entire set of iterative factorial experiments. Normally, interaction effects are computed over a set of $k$ factors in a single experimental design. In this case, interaction effects between all subsets of factors are comparable because they are computed using the same set of system responses, and therefore, are relative to the same scale. However, when system responses are generated over a range of parameter values, interaction effects are computed on different scales, which undermines any comparisons. This issue does not affect main effects, which are absolute measures of the effects of factors on the response and can be compared across the parameter space with no loss of generality.

In order to address this issue concerning interaction effects and facilitate comparisons across the entire parameter space, we introduce the concept of the maximum interaction effect. The maximum interaction effect represents the largest possible interaction effect for a given set of factors, which for a $2 \times 2$ design occurs when improving one factor has the same benefit as improving the other factor or both factors. In terms of the sample design in Table 2, the maximum interaction effect occurs when $R_{2}=R_{3}=R_{4}$. We can insert this expression into Equation 3 and then normalize all interaction effects using the maximum interaction effect for each case to facilitate meaningful comparisons. These two expressions are shown in Equation 4 and Equation 5.

$$
e_{A B}^{\max }=\frac{R_{1}-R_{2}-R_{3}+R_{4}}{2}=\frac{R_{1}-R_{4}}{2}
$$




$$
\hat{e}_{A B}=\frac{e_{A B}}{e_{A B}^{\max }}=\frac{R_{1}-R_{2}-R_{3}+R_{4}}{R_{1}-R_{4}}
$$

\section{RESULTS}

The results of the factorial design experiments are summarized in Figures 1 to 5 . Figure 1 gives the response values at each factor-level combination, shown as data series for each nurse-to-patient ratio level.

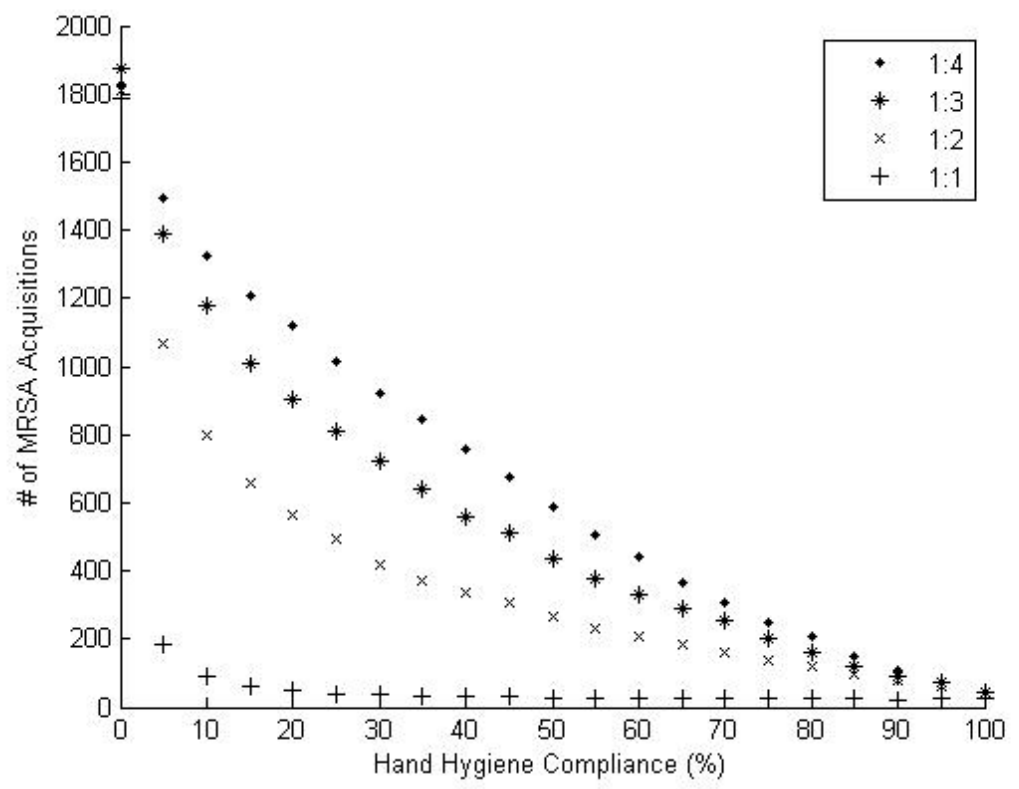

Figure 1: Summary of response values at all factor-level combinations of hand hygiene compliance and nurse-to-patient ratio

Figures 2 to 5 compare the relative effectiveness of the two factors for four different changes in the nurse-to-patient ratio and highlight the interaction between the two factors. There is one figure for each of the investigated changes in nurse-to-patient ratio, namely from 1:4 to 1:3 (Figure 2), 1:3 to 1:2 (Figure 3), 1:2 to 1:1 (Figure 4), and 1:4 to 1:2 (Figure 5). Each series of data points in the plots on the left in Figures 2-5 contains values that represent the difference between the mean main effect of increasing the hand hygiene of nurses and the mean main effect of the corresponding change in the nurse-to-patient ratio. A positive value indicates that the improvement in nurse-to-patient ratio resulted in more prevented MRSA acquisitions than the corresponding increase in hand hygiene compliance. A negative value indicates the improvement in hand hygiene led to a better result. A line is drawn at zero to separate cases where nurse-to-patient ratio performed better from cases when hand hygiene compliance resulted in superior infection control.

Each series contains these difference values for a specified increment in hand hygiene compliance. For example, each series labeled 5\% contains difference values that use the $x$-axis compliance as the minus-level and a hand hygiene compliance level 5\% higher as the plus-level to compute the main effect. Series labeled $25 \%$ use the $x$-axis compliance as the minus-level and a compliance that is $25 \%$ higher as the plus-level. Each series only extends to a compliance value that has $100 \%$ as its maximum plus-level (i.e., $5 \%$ terminates at $95 \%, 10 \%$ at $90 \%$, etc.).

Each data series in the plots on the right in Figures 2-5 represents the set of normalized interaction effects over the entire range of compliance values for a particular increase in the nurse-to-patient ratio. Similar to the difference value figures, each data point represents the normalized interaction effect computed using the baseline compliance level indicated by the $x$-axis, a plus-level specified by the series 
compliance increment, and the increase in nurse-to-patient ratio for that particular figure. Normalized interaction effects have a maximum value of one, which represents the case where the interaction effect is actually maximized. This value is not likely to occur in any experiment, unless one factor has no effect on the response. Interaction effects can also be negative, which represents cases in which improving one factor actually improves the effect of the other factor on the response. However, these cases only occur when compliance increases from very low levels.
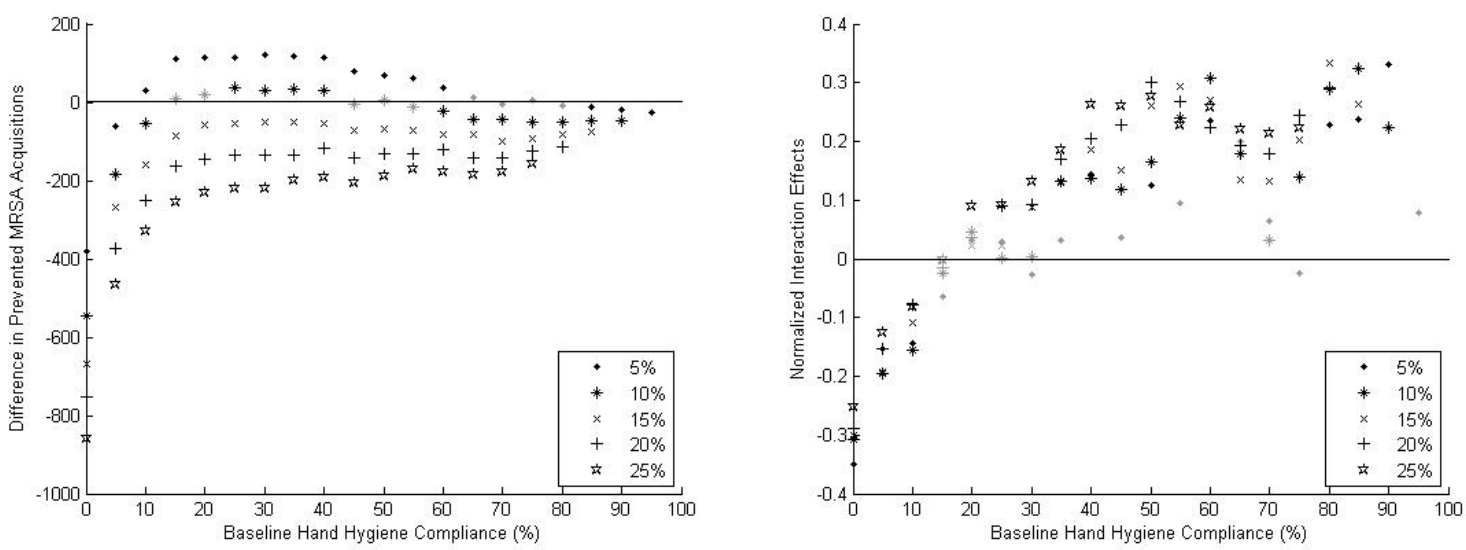

Figure 2: Summary of difference values between hand hygiene compliance main effects and the main effect of increasing the nurse-to-patient ratio from 1:4 to 1:3 (left) and the corresponding normalized interaction effects (right). Non-significant results are shaded in gray $(\alpha=0.05)$.
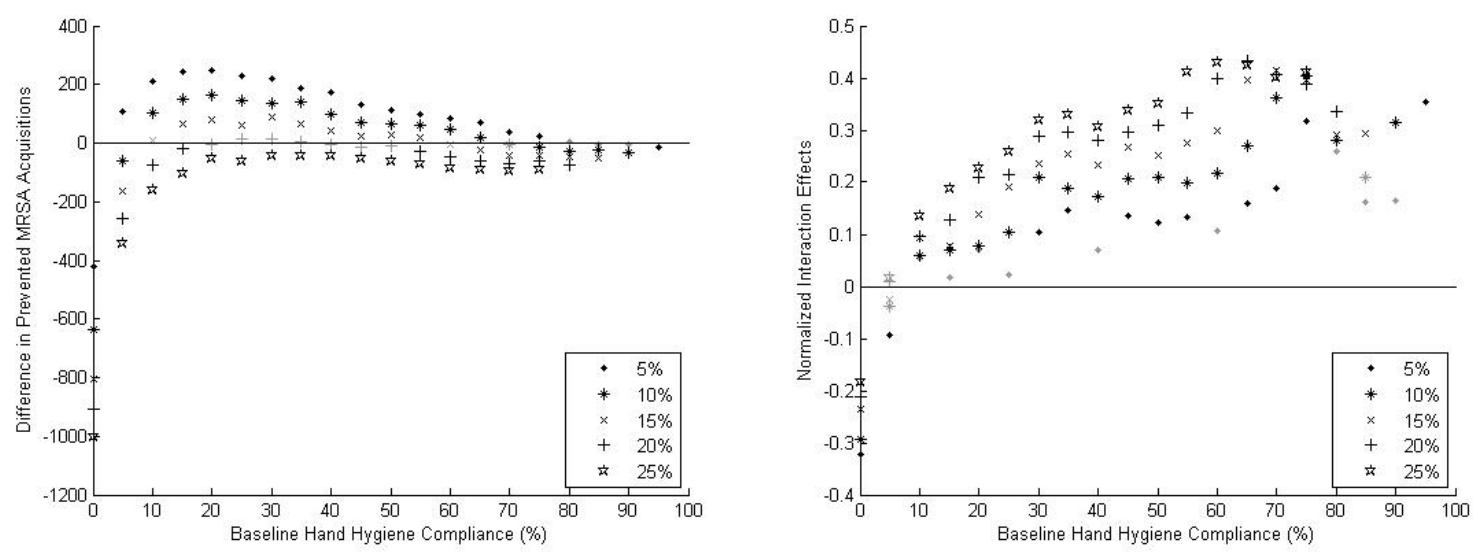

Figure 3: Summary of difference values between hand hygiene compliance main effects and the main effect of increasing the nurse-to-patient ratio from 1:3 to 1:2 (left) and the corresponding normalized interaction effects (right). Non-significant results are shaded in gray $(\alpha=0.05)$.

A two-sided, two-sample T-test with unequal and unknown variances was applied to each main effect difference value, and a two-sided, single-sample T-test with unknown variance was applied to each normalized interaction effect. These hypothesis tests assume that the distributions of main effect difference values and normalized interaction effects are both normal. We are not able to validate this assumption with a goodness of fit test due to our small sample size for each design point. As a consequence of this limitation, we conducted these tests at the $\alpha=0.05$ level to account for some of the uncertainty with the results. Equations for the test statistics and degrees of freedom are summarized in Table 3. Non-significant difference values cannot differentiate between the impact of hand hygiene compliance and the nurse-to- 
patient ratio. Non-significant interaction effects suggest the benefits of improving both factors could be achieved while maximizing the marginal benefit of each factor. Statistical significance is reflected in Figure 2 through Figure 5, in the form of shaded values. Significant results are shaded in black, whereas non-significant results are shaded in gray and typically fall close to zero.

Table 3: Statistical test equations

\begin{tabular}{ccc}
\hline Experimental Output & Test Statistic & Degrees of Freedom \\
\hline Main Effect Difference Value & $T=\frac{\bar{e}_{A}-\bar{e}_{B}}{\sqrt{s_{A}^{2} / n+s_{B}^{2} / n}} \quad v=\frac{\left(s_{A}^{2} / n+s_{B}^{2} / n\right)^{2}}{\left(s_{A}^{2} / n\right)^{2}\left(\frac{\left.s_{B}^{2} / n\right)^{2}}{n-1}\right.}$ \\
Normalized Interaction Effect & $T=\frac{\overline{\hat{e}}_{A B}}{\sqrt{\left(\frac{\left.\hat{s}_{A B}^{2} / n\right)^{2}}{n-1}\right.}}$ & $v=n-1$ \\
\hline
\end{tabular}

* Parameters denoted with a bar above represent sample means. Parameters of the form $s^{2}$ represent sample variances. $n$ is the number of samples (i.e., simulation replications).

As shown in Figure 4, increasing the nurse-to-patient ratio from 1:2 to 1:1 outperforms all considered improvements in hand hygiene compliance. These results are all statistically significant, which suggests this implementation would almost certainly minimize transmission in an ICU setting. We do not provide figures that summarize improvements to a $1: 1$ ratio from ratios such as $1: 3$ or $1: 4$, as these changes are likely to demonstrate an even larger discrepancy between the effects of the two factors. In addition, improvements in hand hygiene compliance from $0 \%$ to higher levels resulted in larger benefits than any associated change in nurse-to-patient ratio. However, it is unlikely that healthcare workers have a baseline compliance of $0 \%$, so this aspect of the figures will not be discussed in additional detail.

In general, the figures show that increasing the nurse-to-patient ratio outperformed small improvements (i.e., those less than 10\%) in hand hygiene compliance, unless the baseline compliance level was extremely high. The benefit of increasing the nurse-to-patient ratio appears to reach a maximum at lower hand hygiene compliance levels, before tapering off and being overtaken by increases in hand hygiene compliance from higher baseline levels. Larger increases in hand hygiene compliance can prevent more MRSA acquisitions at lower baseline compliance levels when nurse-to-patient ratios are relatively low (i.e., $1: 3$ or $1: 4)$.

The interaction effect plots in Figures 2 to 5 highlight some important trends when considering multiple infection control measures. These figures show that interaction effects increase as the baseline hand hygiene compliance level becomes higher, which indicates that there is a diminishing return associated with combining increases in nurse-to-patient ratios with improvements in hand hygiene from high baseline levels. These trends support the conclusions from the main effect figures that hospitals that have been successful at improving hand hygiene compliance can continue to reduce the incidence of MRSA transmission more effectively by further increasing the compliance of healthcare workers. At lower baseline compliance levels, interaction effects are much smaller, which indicates that improvements in compliance can be combined with increases in the nurse-to-patient ratio without losing a significant amount of the potential benefit. Interaction effects for increases to a 1:1 nurse-to-patient ratio are large for any improvement in compliance, and become very large for improvements from high baseline compliance lev- 
els. This trend suggests that if a 1:1 nurse-to-patient ratio is achieved, then hand hygiene compliance becomes a less critical factor with respect to spreading MRSA among patients, assuming productive interactions (i.e., those that result in MRSA transmission) between healthcare workers are minimal.
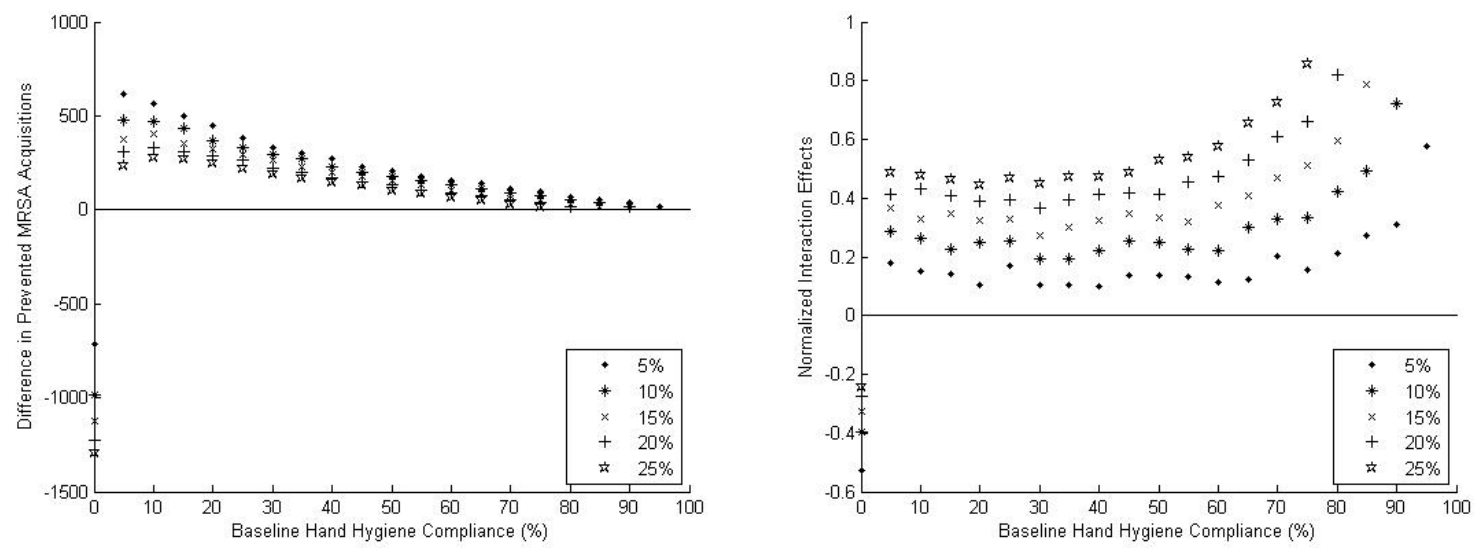

Figure 4: Summary of difference values between hand hygiene compliance main effects and the main effect of increasing the nurse-to-patient ratio from 1:2 to 1:1 (left) and the corresponding normalized interaction effects (right). Non-significant results are shaded in gray $(\alpha=0.05)$.
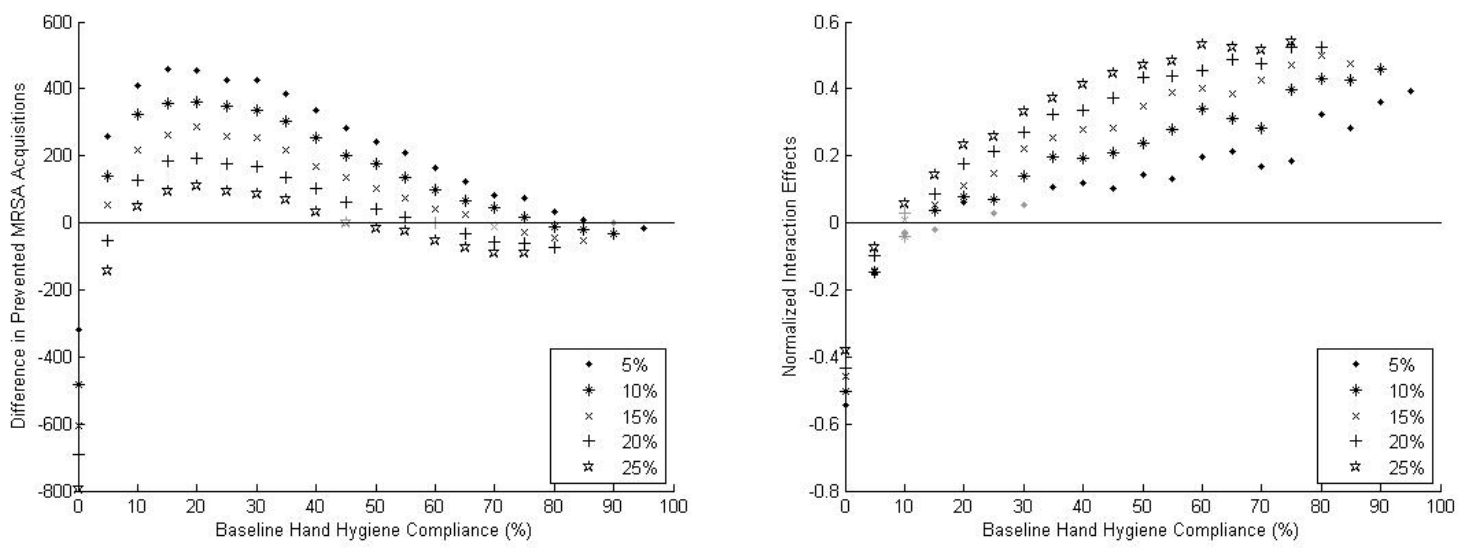

Figure 5: Summary of difference values between hand hygiene compliance main effects and the main effect of increasing the nurse-to-patient ratio from 1:4 to 1:2 (left) and the corresponding normalized interaction effects (right). Non-significant results are shaded in gray $(\alpha=0.05)$.

\section{CONCLUSIONS}

We compared the relative effectiveness of improving the hand hygiene compliance of nurses and increasing the nurse-to-patient ratio by applying a full $2^{k}$ factorial design to the output of an agent-based simulation. Our results suggest that when baseline hand hygiene compliance is low, it may be more effective to hire additional nurses than to rely on improvements in hand hygiene, unless those improvements are very large and are achieved very quickly. At high baseline levels of compliance, it may be more effective to continue focusing on improving compliance than to increase staffing levels.

Previous studies have not quantified the differences between the interventions in detail. This deficiency is addressed by our results in Figures 2 to 5, which compare the performance of the two factors across a wide range of performance levels. In addition, we have provided some indication as to how large 
an improvement in hand hygiene from a given baseline level is required to outperform a change in the nurse-to-patient ratio.

By applying factorial design methods iteratively over a wide range of parameter values, we demonstrated the effectiveness of this methodology for evaluating infection control measures. Main effects have a straightforward application, because they represent the effectiveness of various control measures in reducing MRSA acquisition rates. Interaction effects are also important, because they provide insight with respect to the efficiency of improving multiple control measures simultaneously. Some infection control strategies may seek to maximize effectiveness, whereas others may require more efficiency, and these metrics help to differentiate between the two scenarios. Ideally, with unlimited resources, hospitals would be able to institute multiple interventions aimed at reducing the incidence of hospital-acquired infections. However, hospitals and resident epidemiologists often face limited budgets and thus need to make choices among different interventions.

Our results did not include an analysis of the relative costs associated with each intervention, but this type of analysis could be performed in future studies. At first glance, it would seem less expensive to improve hand hygiene compliance than to hire additional nurses. The average cost of an ICU nurse in the United States is approximately \$62,733 (Salary.com 2010). However, improving hand hygiene and achieving a sustained increase in compliance has proven to be a difficult task, and there is little data to support how much improvement can be attained for a given investment. Many interventions have led to transient increases in hand hygiene compliance, but few studies have been able to demonstrate sustained improvement, which brings into question whether it is possible to achieve a sustained increase in the hand hygiene compliance of healthcare workers (Boyce, Pittet, and Healthcare Infection Control Practices Advisory Committee 2002).

Our results reinforce the importance of basing decisions concerning infection control strategies on individual hospital circumstances, which may assist hospitals in reducing MRSA acquisition rates by taking into account their baseline hand hygiene compliance rates and staffing ratios. There are situations when improving multiple factors is necessary, such as an outbreak, and other situations when improving one factor can reduce MRSA acquisition sufficiently well. Understanding the interaction effects between infection control measures can help determine the best approach. In future work, we may investigate the relative effectiveness of a larger set of infection control measures, which would likely require a more robust experimental design methodology to account for non-linearities in multiple dimensions.

\section{ACKNOWLEDGMENTS}

The authors acknowledge Angela C. Comer, MPH for her assistance with parameter estimation and her careful review of the manuscript. Dr. Golden was supported in part by the Center for Health Information and Decision Systems in the Robert H. Smith School of Business at the University of Maryland. Dr. Wasil was supported in part by a Kogod Research Professorship at American University. Dr. Furuno was supported by National Institutes of Health Career Development Award 1K01AI071015-03. Dr. Harris was supported by National Institutes of Health Midcareer Investigator Award 1K24AI079040.

\section{REFERENCES}

Angus, D. C., W. T. Linde-Zwirble, C. A. Sirio, A. J. Rotondi, L. Chelluri, R. C. Newbold III, J. R. Lave, and M. R. Pinsky. 1996. "The Effect of Managed Care on ICU Length of Stay: Implications for Medicare." Journal of the American Medical Association 276:1075-1082.

Antia, R., and M. Lipsitch. 1997. "Mathematical Models of Parasite Responses to Host Immune Defences.” Parasitology 115 Suppl:S155-67.

Austin, D. J., M. J. Bonten, R. A. Weinstein, S. Slaughter, and R. M. Anderson. 1999. "Vancomycinresistant Enterococci in Intensive-Care Hospital Settings: Transmission Dynamics, Persistence, and the Impact of Infection Control Programs." Proceedings of the National Academy of Sciences USA 96:6908-13. 
Barnes, S., B. Golden, and E. Wasil. 2010. "MRSA Transmission Reduction Using Agent-Based Modeling and Simulation." INFORMS Journal on Computing 22(4):635-646.

Beggs, C. B., C. J. Noakes, S. J. Shepherd, K. G. Kerr, P. A. Sleigh, and K. Banfield. 2006. "The Influence of Nurse Cohorting on Hand Hygiene Effectiveness." American Journal of Infection Control 34:621-626.

Beggs, C. B., S. J. Shepherd, and K. G. Kerr. 2008. "Increasing the Frequency of Hand Washing by Healthcare Workers does not Lead to Commensurate Reductions in Staphylococcal Infection in a Hospital Ward." BMC Infectious Diseases 8:114.

Boyce, J.M., D. Pittet, Healthcare Infection Control Practices Advisory Committee, Society for Healthcare Epidemiology of America, Association for Professionals in Infection Control, and Infectious Diseases Society of America Hand Hygiene Task Force. 2002. "Guideline for Hand Hygiene in Health-Care Settings: Recommendations of the Healthcare Infection Control Practices Advisory Committee and the HICPAC/SHEA/APIC/IDSA Hand Hygiene Task Force." Infection Control and Hospital Epidemiology 23:S3-40.

Cosgrove, S. E., Y. Qi, K. S. Kaye, S. Harbarth, A. W. Karchmer, and Y. Carmeli. 2005. "The Impact of Methicillin Resistance in Staphylococcus Aureus Bacteremia on Patient Outcomes: Mortality, Length of Stay, and Hospital Charges." Infection Control and Hospital Epidemiology 26:166-74.

Cromer, A. L., S. C. Latham, K. G. Bryant, S. Hutsell, L. Gansauer, H. A. Bendyk, R. Steed, and M. C. Carney. 2008. "Monitoring and Feedback of Hand Hygiene Compliance and the Impact on FacilityAcquired Methicillin-Resistant Staphylococcus Aureus." American Journal of Infection Control 36:672-677.

Duggan, J. M., S. Hensley, S. Khuder, T. J. Papadimos, and L. Jacobs. 2008. "Inverse Correlation Between Level of Professional Education and Rate of Handwashing Compliance in a Teaching Hospital." Infection Control and Hospital Epidemiology 29:534-538.

Fridkin, S. K., S. M. Pear, T. H. Williamson, J. N. Galgiani, and W. R. Jarvis. 1996. "The Role of Understaffing in Central Venous Catheter-Associated Bloodstream Infections." Infection Control and Hospital Epidemiology 17:150-158.

Girou, E., S. Loyeau, P. Legrand, F. Oppein, and C. Brun-Buisson. 2002. "Efficacy of Handrubbing with Alcohol Based Solution Versus Standard Handwashing with Antiseptic Soap: Randomised Clinical Trial." BMJ 325:362.

Grundmann, H., S. Hori, B. Winter, A. Tami, and D. J. Austin. 2002. "Risk Factors for the Transmission of Methicillin-Resistant Staphylococcus Aureus in an Adult Intensive Care Unit: Fitting a Model to the Data." Journal of Infectious Diseases 185:481-488.

Honda, H., M. J. Krauss, C. M. Coopersmith, M. H. Kollef, A. M. Richmond, V. J. Fraser, and D. K. Warren. 2010. "Staphylococcus Aureus Nasal Colonization and Subsequent Infection in Intensive Care Unit Patients: Does Methicillin Resistance Matter?" Infection Control and Hospital Epidemiology 31:584-591.

Iwashyna, T. J., A. A. Kramer, and J. M. Kahn. 2009. "Intensive Care Unit Occupancy and Patient Outcomes." Critical Care Medicine 37:1545-1557.

Kirkland, K. B., and J. M. Weinstein. 1999. "Adverse Effects of Contact Isolation." Lancet 354:11771178.

McKenzie, F. E., and E. M. Samba. 2004. "The Role of Mathematical Modeling in Evidence-Based Malaria Control." American Journal of Tropical Medicine and Hygiene 71:94-96.

Moland, E. S., N. D. Hanson, J. A. Black, A. Hossain, W. Song, and K. S. Thomson. 2006. "Prevalence of Newer Beta-Lactamases in Gram-Negative Clinical Isolates Collected in the United States from 2001 to 2002." Journal of Clinical Microbiology 44:3318-24.

Muto, C. A., J. A. Jernigan, B. E. Ostrowsky, H. M. Richet, W. R. Jarvis, J. M. Boyce, and B. M. Farr. 2003. "SHEA Guideline for Preventing Nosocomial Transmission of Multidrug-Resistant Strains of Staphylococcus Aureus and Enterococcus." Infection Control and Hospital Epidemiology 24:362-386. 
Ojajarvi, J. 1980. "Effectiveness of Hand Washing and Disinfection Methods in Removing Transient Bacteria After Patient Nursing." International Journal on Hygiene and Environmental Health 85:193203.

Pittet, D., S. Hugonnet, S. Harbarth, P. Mourouga, V. Sauvan, S. Touveneau, and T. V. Perneger. 2000. "Effectiveness of a Hospital-Wide Programme to Improve Compliance with Hand Hygiene. Infection Control Programme." Lancet 356:1307-12.

Raboud, J., R. Saskin, A. Simor, M. Loeb, K. Green, D. E. Low, and A. McGreer. 2005. "Modeling Transmission of Methicillin-Resistant Staphylococcus Aureus among Patients Admitted to a Hospital." Infection Control and Hospital Epidemiology 26:607-615.

Raboud, J., R. Saskin, K. Wong, C. Moore, G. Parucha, J. Bennett, K. Green, D. Low, M. Loeb, A. Simore, and A. McGreer. 2004. "Patterns of Handwashing Behavior and Visits to Patients on a General Medical Ward of Healthcare Workers." Infection Control and Hospital Epidemiology 25:198-202.

Ridenour, G., R. Lampen, J. Federspiel, S. Kritchevsky, E. Wong, and M. Climo. 2007. "Selective Use of Intranasal Mupirocin and Chlorhexidine Bathing and the Incidence of Methicillin-Resistant Staphylococcus Aureus Colonization and Infection Among Intensive Care Unit Patients." Infection Control and Hospital Epidemiology 28:1155-1161.

Salary.com. Career Advancement Tools and Resources. Accessed July 13, 2010. http://swz.salary.com/salarywizard/layouthtmls/swzl_compresult_national_HC07000274.html.

Sebille, V., S. Chevret, and A. J. Valleron. 1997. "Modeling the Spread of Resistant Nosocomial Pathogens in an Intensive- Care Unit." Infection Control and Hospital Epidemiology 18:84-92.

Warren, D. K., R. M. Guth, C. M. Coopersmith, L. R. Merz, J. E. Zack, and V. J. Fraser. 2006. "Epidemiology of Methicillin-Resistant Staphylococcus Aureus Colonization in a Surgical Intensive Care Unit." Infection Control and Hospital Epidemiology 27:1032-1040.

Weber, S. G., S. S. Huang, S. Oriola, W. C. Huskins, G. A. Noskin, K. Harriman, R. N. Olmsted, M. Bonten, T. Lundstrom, M. W. Climo, M. Roghmann, C. L. Murphy, and T. B. Karchmer. 2007. "Legislative Mandates for Use of Active Surveillance Cultures to Screen for Methicillin-Resistant Staphylococcus Aureus and Vancomycin-Resistant Enterococci: Position Statement from the Joint SHEA and APIC Task Force." Infection Control and Hospital Epidemiology 28:249-260.

\section{AUTHOR BIOGRAPHIES}

SEAN BARNES is a doctoral candidate in Applied Mathematics and Scientific Computation in the Department of Mathematics at the University of Maryland, College Park. He received his undergraduate and master's degrees in aerospace engineering from the Georgia Institute of Technology in 2006 and 2007 respectively. His research interests include agent-based modeling and simulation, health care applications, and complex systems. His email address is sbarnes@math.umd.edu.

BRUCE GOLDEN is the France-Merrick Chair in Management Science in the Robert H. Smith School of Business at the University of Maryland, College Park. Bruce Golden received his undergraduate degree in mathematics from the University of Pennsylvania and his master's and doctoral degrees from the Massachusetts Institute of Technology. He joined the faculty of the University of Maryland Business School in 1976 and served as a Department Chairman from 1980 to 1996. Since 1999, Bruce has served as Editor-in-Chief of Networks. Before that, he was Editor-in-Chief of the INFORMS Journal on Computing. His research interests include heuristic search, combinatorial optimization, networks, and healthcare operations management. His email address is bgolden@rhsmith.umd.edu.

EDWARD WASIL is a professor of management science in the Kogod School of Business at American University. He received his doctoral degree from the University of Maryland, College Park. He serves as the feature article editor of the INFORMS Journal on Computing and the associate editor of INFOR: Canadian Journal of Operational Research and Information Processing. His research focuses on network 
optimization and applications of metaheuristics to optimization problems. He was the American University Scholar/Teacher of the year in 2006. His email address is ewasil@american.edu.

JON FURUNO is an assistant professor of epidemiology and public health at the University of Maryland School of Medicine in Baltimore, MD. He is also the Director of the Ph.D. track in Molecular Epidemiology. He received his undergraduate degree from the University of Rhode Island and his master's and doctoral degrees in Epidemiology from the University of Maryland. His research interests focus on multiple topics related to epidemiology of infectious diseases in healthcare settings, as well as improving healthcare quality and associated patient outcomes. His email address is jfuruno@epi.umaryland.edu.

ANTHONY HARRIS is a professor of epidemiology and public health and division head at the University of Maryland School of Medicine. He received his undergraduate and medical degrees from McGill University and an Master's Degree in Public Health from Harvard University. His research interests include emerging pathogens, antimicrobial-resistant bacteria, infection control interventions, epidemiologic methods in infectious diseases and medical informatics. He has published over 100 papers and has funding from the NIH, CDC and AHRQ to study antibiotic resistance and hospital epidemiology. His email address is aharris@epi.umaryland.edu. 